\title{
A new strategy of promoting cisplatin chemotherapeutic efficiency by targeting endoplasmic reticulum stress (Review)
}

\author{
YE XU ${ }^{1,2}$, CHUNYAN WANG ${ }^{1}$ and ZHIXIN $\mathrm{LI}^{2}$ \\ ${ }^{1}$ Medical Research Laboratory and ${ }^{2}$ Department of Histology and Embryology, \\ Jilin Medical College, Jilin, Jilin 132013, P.R. China
}

Received May 14,2013; Accepted August 12, 2013

DOI: $10.3892 / \mathrm{mco} .2013 .202$

\begin{abstract}
Cisplatin (cis-diamminedichloroplatinum II, CDDP) is one of the most effective chemotherapeutic agents and is widely used in the treatment of solid tumors. However, its side effects and acquired resistance gained during the course of treatment may limit its usage. It is generally considered to be a cytotoxic drug that kills cancer cells by damaging their DNA and inhibiting DNA synthesis to induce apoptosis via the mitochondrial death pathway or through plasma membrane disruption, triggering the Fas death receptor pathway. The endoplasmic reticulum (ER) is one of the most important protein-folding compartments within the cell and an intracellular $\mathrm{Ca}^{2+}$ storage organelle. The ER contains a number of molecular chaperones, which may play an important role in determining cellular sensitivity to ER stress and apoptosis. The aim of this review was to summarize our current understanding regarding the mechanisms of ER stress response by which cisplatin induces cell death and the basis for cisplatin resistance. Various aspects were addressed, including the two-way regulation of ER stress, the involvement of ER stress in cisplatin-induced cell death and drug resistance and the drugs enhancing cisplatin-induced cell death by interfering with ER stress. An understanding of how ER stress signaling pathways regulate cisplatin-induced cell death may enable the development of more effective therapeutic strategies for the treatment of cancer.
\end{abstract}

\section{Contents}

1. Introduction

2. ER stress has a two-way regulation of maintaining cell survival or triggering cell death

3. ER stress is involved in cisplatin-induced cell death and is associated with its toxicity and side effects

Correspondence to: Professor Zhixin Li, Department of Histology and Embryology, Jilin Medical College, 5 Jilin Street, Jilin, Jilin 132013, P.R. China

E-mail:1zx-62@163.com

Key words: cisplatin, chemotherapy, endoplasmic reticulum stress
4. The prosurvival role of ER stress causes cisplatin drug resistance

5. Interference of ER stress may enhance cisplatin-induced tumor cell death

6. Conclusions

\section{Introduction}

Cisplatin (cis-diamminedichloroplatinum II, CDDP) is one of the most effective chemotherapeutic agents and is widely used in the treatment of solid tumors. However, the side effects and drug resistance during the course of the treatment may limit its usage (1-3). It is generally considered as a cytotoxic drug that kills cancer cells by damaging their DNA and inhibiting DNA synthesis. Cisplatin-induced DNA damage activates various signaling pathways to prevent or promote cell death, predominantly via apoptosis (4). It was recently demonstrated that cisplatin may induce endoplasmic reticulum stress (ER stress) and non-nucleus-dependent apoptotic signal activation (5-7). Currently, the ER is considered to be involved in cisplatin-induced tumor cell death as a cell stress signaling receptor. The aim of this review was to investigate the mechanisms of ER stress during cisplatin chemotherapy and assess the possibility of promoting the chemotherapeutic effects of cisplatin by targeting ER stress.

2. ER stress has a two-way regulation of maintaining cell survival or triggering cell death

The ER is an organelle with crucial biosynthetic and signaling functions in eukaryotic cells. These processes are facilitated and monitored by several resident chaperone molecules and $\mathrm{Ca}^{2+}$-binding proteins, including glucose-regulated proteins, such as $78 \mathrm{kDa}$ glucose-regulated protein (GRP78) or immunoglobulin heavy-chain-binding protein (BiP), calreticulin and calnexin, as well as several folding enzymes, such as the thioredoxin-like protein disulfide isomerase (PDI). Various physiological and pathological conditions, including hypoxia, ER $\mathrm{Ca}^{2+}$ depletion, oxidative injury, high-fat diet, hypoglycemia and viral infections, may cause an imbalance between ER protein-folding load and capacity, leading to the accumulation of unfolded proteins in the ER lumen and resulting in 'ER stress'. ER stress is a key reaction in the cell's 
response to environmental factors and triggers the unfolded protein reaction (UPR); UPR involves the ER molecular chaperone GRP78/BiP, the ER stress sensor protein PKR-like ER kinase (PERK), the inositol-requiring enzyme 1 (IRE-1) and the activating transcription factor 6 (ATF6), as well as their downstream signaling pathways (8).

A moderate UPR activation possesses an anti-apoptotic role that enhances tumor cell survival and imparts drug resistance; however, severe UPR activation leads to apoptosis. The IRE-1 and PERK pathways in the UPR system are key signal transduction pathways in ER stress-induced cell autophagy, apoptosis and in complicated regulatory networks (9). UPR in solid tumors inhibits the majority of translation processes, reduces the processing protein burden in the ER and upregulates the molecular chaperones GRP78/BiP and GRP94, in order to increase the ER protein-folding capacity by the PERK/eukaryotic translation initiation factor $2 \alpha$ (eIF2 $\alpha$ ), IRE-1 and activating transcription factor 6 (ATF6) pathways. Eventually, proteins that are not correctly folded are degraded by relevant protein degradation pathways (proteasome and autophagy), or induce cell apoptosis by activating downstream apoptotic signaling molecules, such as CCAAT/enhancer-binding protein homologous protein (CHOP)/GADD153, c-Jun N-terminal kinase (JNK), caspases and the Bcl-2 family (10). IRE-1 may be combined with TNF receptor-associated factor 2 (TRAF2) during ER stress activation, which enhances JNK signal transduction activation. Initially, JNK activation triggers cell autophagy and continuous activation of JNK leads to irreversible cell apoptosis. While eIF $2 \alpha$ in the PERK signal transduction pathway is phosphorylated, the expression of autophagy-related protein (Atg) 12 increases with Atg5-Atg12-Atg16 complex formation, which activates microtubule-associated protein 1 light chain 3 translocation to induce autophagy. PERK may also induce apoptosis by upregulating ATF4 and CHOP to activate the caspase cascade reaction (11).

A number of components that are involved in the execution of ER stress enhance cell survival and also trigger cell death. A common characteristic of these components is possessing regulatory functions involving several signal transduction pathways (12-14). The primary role of the ER stress response is to protect cells under stress by reestablishing homeostasis or attenuating damaging effects. The key regulator of this reverse procedure is GRP78, which is a calcium-binding protein that is mainly located in the ER. GRP78 is a chaperone molecule in protein-folding, which regulates three principle signaling pathways from transmembrane proteins of the ER (15). It is difficult to detect GRP78 in normal cells, as it is usually expressed following stimulation of ER stress. However, sustained high levels of GRP78 may be detected in several tumors $(16,17)$. Clinical studies demonstrated that the expression of GRP78 is upregulated in numerous types of malignant tumors, including lung, hepatic and breast cancer. Empirical studies proved that GRP78 is able to maintain ER calcium homeostasis, inhibit caspase-7 activation, bind to ER-targeting cell apoptosis protein BIK and prevent its activation, and thus inhibit chemotherapeutic-induced apoptosis through several pathways, causing chemotherapeutic resistance in tumors $(18,19)$. This phenotype indicates that tumor cells continually execute responses against chronic stress of harmful conditions and that metabolic changes in tumor cells may result in sustained changes (17). GRP78 inhibits proapoptotic pathways and its increased expression in tumor cells enhances chemotherapeutic resistance, which may be associated with an unfavorable prognosis (15). Wang et al (20) observed an association between high expression of GRP78 and resistance to etoposide, adriamycin, vincristine and topotecan in lung cancer cells, indicating that GRP78 is crucial in regulating chemotherapeutic potency and drug resistance (20).

The sustaining ability of the ER stress response is not limitless. When ER stress becomes more severe, this system switches to proapoptotic regulation, which triggers cell death even in the presence of high levels of GRP78. The key factor in this phenomenon is the transcription factor CHOP. The increased expression of $\mathrm{CHOP}$ induces the transcription of various genes to induce activation of the proapoptotic process, which involves inhibition of Bcl-2 and stimulation of death receptor 5 , activation of caspases, integration of mitochondrial events and amplification of death signals (21).

\section{ER stress is involved in cisplatin-induced cell death and is associated with its toxicity and side effects}

Cisplatin may induce apoptosis via ER stress signaling. It was reported that cisplatin induced apoptotic signaling independently of DNA damage in enucleated cells. In these cells, cisplatin-induced caspase-3 activation requires $\mathrm{Ca}^{2+}$ and $\mathrm{Ca}^{2+}$-dependent calpain protease. The activation of calpain was associated with ER stress, indicating that ER was the cytoplasmic target organelle of cisplatin. In intact cells, cisplatin was shown to induce calpain-dependent activation of the ER-specific caspase-12 and upregulate the expression of another ER stress marker, GRP78 (5). Another study demonstrated similar results, in which the cleavage of procaspase-12 led to the activation of caspase- 9 and caspase- 3 in cisplatin-treated LLC-PK1 cells. In that same study, pretreatment with caspase- 9 inhibitors did not decrease the activation of caspase- 3 and exerted no obvious protective effects. However, treatment with anti-caspase-12 antibody significantly decreased cisplatin-induced apoptosis, indicating that caspase-12 plays an important role in cisplatin-induced apoptosis (6). Cisplatin was also shown to induce apoptosis in enucleated mouse kidney proximal tubule cells (22). The authors of that study observed that cisplatin may induce cell death by cytoplasmic signaling, which was independent of the regulation of the nucleus, but partially regulated by the cyclin-dependent kinase 2 (Cdk2)-E2F1 pathway. The cytoplasmic locations of $\mathrm{Cdk} 2$ are the ER and the Golgi complex. Specific inhibition of Cdk2 blocked ER stress and provided protection. Those findings demonstrated that the cytotoxicity of cisplatin may be precipitated by cytoplasmic events and that cytoplasmic Cdk2 is crucial in cisplatin-induced apoptotic signaling.

The upregulation of the ER stress marker GRP78 was reported to be associated with the sensitivity to platinum-class drugs. Exposure to 2-deoxyglucose, which induces the upregulation of UPR/GRP78, followed by cisplatin treatment, demonstrated that cisplatin enhanced the mitochondria-mediated apoptosis cascade through the activation of caspase- 2 and the downregulation of the expression of DNA damage-repairing 
genes (23). In our previous studies, we demonstrated that cisplatin treatment induced significant ER stress, upregulation of GRP78, PDI and CHOP and activation of caspase-4 in various cancer cell lines, indicating that cisplatin may induce apoptosis through the ER stress pathway $(24,25)$.

ER stress is involved in cisplatin nephrotoxicity, ototoxicity and myocardial damage. Following cisplatin treatment, the expression of the ER stress markers X-box binding protein 1, GRP78 and GRP94 was upregulated and activation of the ER-mediated cell death markers caspase-12 and calpain was observed in rat kidney tissue. Furthermore, the expression of the cleavage products of caspase-12 were increased in vivo. Those results demonstrated that cisplatin nephrotoxicity is associated with ER stress and ER-mediated cell death markers (26). Pre-activation of ER stress exerted a protective effect following cisplatin administration in various renal cell lines. It was demonstrated that pretreatment with tunicamycin (TUNI) or oxidized dithiothreitol significantly decreased cisplatin cytotoxicity, indicating that ER stress pre-activation provides protection against cisplatin nephrotoxicity in various cell lines, although there were differences in quality and quantity among the cell lines (27). It was reported that the activation of eIF2 $\alpha$, which is downstream of the ER stress apoptotic pathway and regulates ATF4, CHOP and caspase-12, -9 and -3 , may be involved in cisplatin-induced nephrotoxicity (28). Injection of recombinant human erythropoietin (rHuEPO) was shown to enhance the recovery from cisplatin-induced acute kidney injury (AKI) in rats by relieving renal functional impairment and exerting significant anti-apoptotic effects. However, rHuEPO inhibited cisplatin-induced AKI through a mechanism possibly involving phosphatidylinositol-3 kinase/Akt activation and inhibition of ER stress-mediated apoptosis (29).

It was demonstrated that cisplatin ototoxicity is also associated with ER stress proteins. In cochlear cells obtained from P3 rats, the two-dimensional difference gel electrophoresis and the matrix-assisted laser desorption time-of-flight analysis demonstrated that the expression of the cisplatin-induced heat shock $70 \mathrm{kDa}$ protein 5 (HSPA5, GRP78), an ER molecular chaperone participating in the ER stress response, was decreased by 1.7 -fold. These changes were concordant with the phosphorylation of GRP58, another ER stress-induced protein (30).

Treatment of C57 mice with cisplatin led to aberrations in myocardial contraction reflected by a decrease in the left ventricular developed pressure (LVDP) and the first derivative of LVDP $( \pm \mathrm{dP} / \mathrm{dt})$. Furthermore, that study revealed that cisplatin treatment induced the ER stress response, the caspase-3 activity was increased and the mitochondrial ultrastructure was altered. Those results indicated that cisplatin is associated with cardiovascular toxicity, which is associated with mitochondrial dysfunction, ER stress and apoptosis (31).

\section{The prosurvival role of ER stress causes cisplatin drug resistance}

The H460et and A549et human lung cancer cell lines were reported to be ER stress-tolerant. Moreover, these cells are cisplatin-resistant. Compared to the parental cells, H460et and
A549et cells led to significant GRP78 upregulation and high levels of phospho-Akt, indicating that cisplatin resistance was associated with ER stress and Akt activation (32). It was also reported that gene silencing of $\mathrm{N}$-ethylmaleimide-sensitive factor attachment protein $\alpha$ (NAPA), which is involved in protein transfer in the ER, rendered tumor cells sensitive to cisplatin and overcame drug resistance in vitro and in vivo (33). By contrast, the overexpression of NAPA increased cisplatin resistance by downregulating cisplatin-induced ER stress and apoptosis.

It was reported that ER stress induction significantly decreased the cisplatin-induced apoptotic rate in gastric cancer cells. The induction of ER stress activated p38 and the inhibition of $\mathrm{p} 38$ hampered the apoptosis tolerance mediated by cisplatin-induced ER stress (34). Namely, cisplatin resistance was obtained through the ER stress response in gastric cancer cells and this resistance was overcome by p38 activity inhibition. That finding indicated that ER stress induced by cisplatin exerts a protective effect against apoptosis through p38 mitogen-activated protein kinase (MAPK) signaling. In hepatocellular carcinoma cells, the UPR triggered by ER stress was shown to inhibit cisplatin-induced apoptosis (35). Furthermore, moderate ER stress pre-activation of these cells inhibited their sensitivity to cisplatin-induced apoptosis.

We recently demonstrated a critical role for the ubiquitin-binding protein p62/SQSTM1 in cisplatin resistance in human ovarian cancer cells (HOCCs) (25). Specifically, we observed that cisplatin-resistant SKOV3/DDP cells expressed significantly higher levels of p62 compared to those expressed by cisplatin-sensitive SKOV3 cells. In SKOV3/DDP cells, p62 binds ubiquitinated proteins for transport to autophagic degradation, reducing apoptosis induced by ER stress. The knockdown of p62 or inhibition of autophagy using 3-methyladenine (3-MA) was shown to resensitize SKOV3/DDP cells to cisplatin. Our data indicated that $\mathrm{p} 62$ acts as a receptor or an adaptor for autophagic degradation of ubiquitinated proteins and plays an important role in preventing ER stress-induced apoptosis, leading to cisplatin resistance in HOCCs.

\section{Interference of ER stress may enhance cisplatin-induced tumor cell death}

Over the last few years, it was demonstrated that certain drugs may enhance the cell-killing effect of cisplatin by interfering with ER stress. Several of these drugs are listed below.

O6-benzylguanine (O6-BG) enhanced cisplatin cytotoxicity and apoptosis in SKOV3x ovarian cancer cells and head and neck cancer cell lines. It was demonstrated that cisplatin combined with O6-BG affects two targets: DNA and ER. O6-BG enhanced cisplatin cytotoxicity and O6-BG treatment leads to more severe cisplatin-induced DNA damage. The evaluation of the effect of cisplatin treatment on ER revealed an augmentation of caspase-12 cleavage in SQ20b and SKOV3x cells. GADD153, an ER stress response gene, was upregulated following a combination treatment with cisplatin and O6-BG, compared to cisplatin alone in SQ20b and SKOV3x cells. ER stress-induced apoptosis was one of the mechanisms underlying O6-BG-enhanced cisplatin activity. In SQ20b cells, treatment with salubrinal, which inhibits ER stress, or with GADD153 small interfering RNA, eliminated 
O6-BG-enhanced cisplatin cytotoxicity and apoptosis through the attenuated cleavage of caspase- 3 and caspase-12. Those data indicated that GADD153 upregulation plays a significant role in O6-BG-enhanced cisplatin cytotoxicity and apoptosis (36).

Bortezomib (PS-341, Velcade) is a proteasome-selective inhibitor and is currently only used for experimental therapy of solid malignant tumors. It was demonstrated that bortezomib induced ER stress and simultaneously inhibited UPR in a pancreatic cancer cell model. Furthermore, that study demonstrated that bortezomib enhanced classic ER stress inducers (TUNI and thapsigargin) that trigger apoptosis in a JNK-dependent manner. Furthermore, that study demonstrated that cisplatin stimulates ER stress and, in combination with bortezomib, leads to increased ER dilation, increased intracellular $\mathrm{Ca}^{2+}$ levels and cell death. Bortezomib combined with cisplatin treatment induced JNK activation and apoptosis, resulting in bortezomib-enhanced pancreatic cancer cell sensitivity to ER stress-induced apoptosis. Bortezomib potently enhanced the cisplatin antitumor activity (37).

Compared to the ER stress inducer thapsigargin, the histone acetyltransferase inhibitor suberoylanilide hydroxamic acid (SAHA) was shown to enhance cisplatin-induced apoptosis in oral squamous cell cancer (OSCC) cells via ER stress. SAHA increased cisplatin cytotoxicity through ER stress-induced apoptosis. Cisplatin/SAHA treatment effectively induced apoptosis in HSC-3 cells (OSCC cell line), with a significant increase in caspase- 4 and caspase- 12 activity. SAHA treatment alone rapidly induced sustained eIF $2 \alpha$ phosphorylation. The eIF2 $\alpha$ dephosphorylation inhibitor salubrinal inhibited ER stress, which eliminated SAHA-enhanced cisplatin cytotoxicity. The level of phosphorylated Akt was decreased in SAHA-treated cells, which correlated with an increase in the activity of protein phosphatase 1 (PP1). Those results indicated that the upregulation of ER stress-specific events is one of the mechanisms of SAHA-enhanced cisplatin-induced apoptosis and that PP1 upregulation leading to Akt dephosphorylation plays an important role in SAHA-enhanced cisplatin-induced apoptosis (38).

Cryptotanshinone, which has been identified as an effective ER stress inducer leading to apoptosis in various tumor cell lines, including HepG2 hepatic cancer and MCF7 breast cancer cell lines, enhances cisplatin cytotoxicity by promoting ER stress-induced apoptosis. MAPKs function as mediators in this process. Reactive oxygen species generated by cryptotanshinone play an important role in ER stress-induced apoptosis. Cryptotanshinone may enhance the effect of certain anticancer drugs, including cisplatin, through ER stress (39).

Moreover, we demonstrated that cisplatin treatment induces ER stress, apoptosis and autophagy in HeLa human cervical cancer cells. Autophagy efficiently transports cisplatin-induced misfolded proteins for degradation, allowing cells to escape ER stress-mediated apoptosis and the mitochondrial apoptotic pathway, thus maintaining cell homeostasis and survival. The inhibition of autophagy using 3-MA or chloroquine increased intracellular misfolded proteins, which enhanced cellular apoptosis. Furthermore, we observed that the ER stress inducer TUNI augmented cisplatin cytotoxicity by increasing ER stress-mediated apoptosis. In addition, autophagy blockage or ER stress elevation increased the sensitivity of HeLa cells to cisplatin. Autophagy inhibition or ER stress induction may represent therapeutic targets for the improvement of cisplatin efficacy (24).

It was recently demonstrated that ER stress signaling regulates the switch between autophagy and apoptosis in cisplatin treatment. The use of multi-disciplinary methods to investigate the correlation between autophagy and apoptosis in NRE-52E kidney cells treated with cisplatin revealed that there are two cisplatin-sensitive thresholds determining the occurrence of autophagy or apoptosis: $10 \mu \mathrm{M}$ of cisplatin activated autophagy, maintaining cell survival, whereas 3-MA treatment simultaneously affected cell viability and induced apoptosis. On the contrary, $50 \mu \mathrm{M}$ of cisplatin led to apoptosis and cell death. Pretreatment with taurine rescued cells by delaying apoptosis and sustaining autophagy. Therefore, autophagy protects NRK-52E cells from cisplatin injury. The investigation of ER-specific markers, such as GRP78, GRP94 and GADD153/CHOP revealed that ER stress signaling may play a central role in the crosstalk between cisplatin-induced autophagy and apoptosis (40).

\section{Conclusions}

The regulation of survival promotion and death induction through ER stress signaling during cisplatin treatment in tumor cells was previously demonstrated. Based on those data, several research teams proceeded to experimental therapeutic studies and acquired primary results, which indicated the potential application of this research field in tumor chemotherapy. Investigators are currently attempting to increase the chemotherapeutic effect or overcome the resistance to cisplatin by deciphering the complex network of ER stress. The in-depth comprehension of the precise mechanism underlying the regulation of autophagy and apoptosis through ER stress response signaling in different cells treated with cisplatin may help enhance the clinical effect of cisplatin, reduce the side effects and overcome therapeutic drug resistance, establishing a novel application of this traditional chemotherapeutic agent.

\section{Acknowledgements}

The present study was supported by the National Natural Science Foundation of China (nos. 81372793 and 81100808), the Natural Science Foundation of Jilin Province (no. 201015240) and the Department of Education of Jilin Province Project (no. 2013361).

\section{References}

1. Loehrer PJ and Einhorn LH: Drugs five years later: cisplatin. Ann Int Med 100: 704-713, 1984.

2. Reedijk J: New clues for platinum antitumor chemistry: kinetically controlled metal binding to DNA. Proc Natl Acad Sci USA 100: 3611-3616, 2003.

3. Woźniak K and Błasiak J: Recognition and repair of DNA-cisplatin adducts. Acta Biochim Pol 49: 583-596, 2002.

4. Basu A and Krishnamurthy S: Cellular responses to cisplatin-induced DNA damage. J Nucleic Acids 2010: 201367, 2010.

5. Mandic A, Hansson J, Linder S and Shoshan MC: Cisplatin induces endoplasmic reticulum stress and nucleus-independent apoptotic signaling. J Biol Chem 278: 9100-9106, 2003.

6. Yu F, Megyesi J and Price PM: Cytoplasmic initiation of cisplatin cytotoxicity. Am J Physiol Renal Physiol 295: F44-F52, 2008. 
7. Martins I, Kepp O, Schlemmer F, Adjemian S, Tailler M, Shen S, Michaud M, Menger L, Gdoura A, Tajeddine N, Tesniere A, Zitvogel L and Kroemer G: Restoration of the immunogenicity of cisplatin-induced cancer cell death by endoplasmic reticulum stress. Oncogene 30: 1147-1158, 2011.

8. Gorman AM, Healy SJ, Jäger R and Samali A: Stress management at the ER: regulators of ER stress-induced apoptosis. Pharmacol Ther 134: 306-316, 2012.

9. Verfaillie T, Salazar M, Velasco G and Agostinis P: Linking ER stress to autophagy: potential implications for cancer therapy. Int J Cell Biol 2010: 930509, 2010.

10. Rodriguez D, Rojas-Rivera D and Hetz C: Integrating stress signals at the endoplasmic reticulum: the $\mathrm{BCL}-2$ protein family rheostat. Biochim Biophys Acta 1813: 564-574, 2011.

11. Heath-Engel HM, Chang NC and Shore GC: The endoplasmic reticulum in apoptosis and autophagy: role of the BCL-2 protein family. Oncogene 27: 6419-6433, 2008.

12. Boyce M and Yuan J: Cellular response to endoplasmic reticulum stress: a matter of life or death. Cell Death Differ 13: 363-373, 2006.

13. Marciniak SJ and Ron D: Endoplasmic reticulum stress signaling in disease. Physiol Rev 86: 1133-1149, 2006.

14. Wu J and Kaufman RJ: From acute ER stress to physiological roles of the unfolded protein response. Cell Death Differ 13: 374-384, 2006.

15. Lee AS: GRP78 induction in cancer: therapeutic and prognostic implications. Cancer Res 67: 3496-3499, 2007.

16. Pyrko P, Schönthal AH, Hofman FM, Chen TC and Lee AS: The unfolded protein response regulator GRP78/BiP as a novel target for increasing chemosensitivity in malignant gliomas. Cancer Res 67: 9809-9816, 2007.

17. Christofk HR, Vander Heiden MG, Harris MH, Ramanathan A, Gerszten RE, Wei R, Fleming MD, Schreiber SL and Cantley LC: The M2 splice isoform of pyruvate kinase is important for cancer metabolism and tumour growth. Nature 452: 230-233, 2008.

18. Ranganathan AC, Zhang L, Adam AP and Aguirre-Ghiso JA Functional coupling of $\mathrm{p} 38$-induced up-regulation of $\mathrm{BiP}$ and activation of RNA-dependent protein kinase-like endoplasmic reticulum kinase to drug resistance of dormant carcinoma cells Cancer Res 66: 1702-1711,2006.

19. Li J and Lee AS: Stress induction of GRP78/BiP and its role in cancer. Curr Mol Med 6: 45-54, 2006.

20. Wang L, Wang Q, Wang Z, Yu Z, Wang J, Xiong H, Yin Q, Wang Y, Wang T and Tong S: Relationship between chemosensitivity in vitro and the expression of GRP78 in non-small cell lung cancer. Chin J Lung Canc 9: 483-487, 2006 (In Chinese).

21. Oyadomari S and Mori M: Roles of CHOP/GADD153 in endoplasmic reticulum stress. Cell Death Differ 11: 381-389, 2004.

22. Yu F, Megyesi J, Safirstein RL and Price PM: Involvement of the CDK2-E2F1 pathway in cisplatin cytotoxicity in vitro and in vivo. Am J Physiol Renal Physiol 293: F52-F59, 2007.

23. Gaddameedhi S and Chatterjee S: Association between the unfolded protein response, induced by 2 -deoxyglucose, and hypersensitivity to cisplatin: a mechanistic study employing molecular genomics. J Cancer Res Ther Suppl 1: S61-S66, 2009

24. Xu Y, Yu H, Qin H, Kang J, Yu C, Zhong J, Su J, Li H and Sun L: Inhibition of autophagy enhances cisplatin cytotoxicity through endoplasmic reticulum stress in human cervical cancer cells. Cancer Lett 314: 232-243, 2012.

25. Yu H, Su J, Xu Y, Kang J, Li H, Zhang L, Yi H, Xiang X, Liu F and Sun L: p62/SQSTM1 involved in cisplatin resistance in human ovarian cancer cells by clearing ubiquitinated proteins Eur J Cancer 47: 1585-1594, 2011.
26. Peyrou M, Hanna PE and Cribb AE: Cisplatin, gentamicin, and p-aminophenol induce markers of endoplasmic reticulum stress in the rat kidneys. Toxicol Sci 99: 346-353, 2007.

27. Peyrou M and Cribb AE: Effect of endoplasmic reticulum stress preconditioning on cytotoxicity of clinically relevant nephrotoxins in renal cell lines. Toxicol In Vitro 21: 878-886, 2007.

28. Wu CT, Sheu ML, Tsai KS, Chiang CK and Liu SH: Salubrinal, an eIF2 $\alpha$ dephosphorylation inhibitor, enhances cisplatin-induced oxidative stress and nephrotoxicity in a mouse model. Free Radic Biol Med 51: 671-680, 2011.

29. Kong D, Zhuo L, Gao C, Shi S, Wang N, Huang Z, Li W and Hao L: Erythropoietin protects against cisplatin-induced nephrotoxicity by attenuating endoplasmic reticulum stress-induced apoptosis. J Nephrol 26: 219-227, 2013.

30. Coling DE, Ding D, Young R, Lis M, Stofko E, Blumenthal KM and Salvi RJ: Proteomic analysis of cisplatin-induced cochlear damage: methods and early changes in protein expression. Hear Res 226: 140-156, 2007.

31. Ma H, Jones KR, Guo R, Xu P, Shen Y and Ren J: Cisplatin compromises myocardial contractile function and mitochondrial ultrastructure: role of endoplasmic reticulum stress. Clin Exp Pharmacol Physiol 37: 460-465, 2010.

32. Lin Y, Wang Z, Liu L and Chen L: Akt is the downstream target of GRP78 in mediating cisplatin resistance in ER stress-tolerant human lung cancer cells. Lung Cancer 71: 291-297, 2011.

33. Wu ZZ and Chao CC: Knockdown of NAPA using short-hairpin RNA sensitizes cancer cells to cisplatin: implications to overcome chemoresistance. Biochem Pharmacol 80: 827-837, 2010.

34. Feng R, Zhai WL, Yang HY, Jin H and Zhang QX: Induction of ER stress protects gastric cancer cells against apoptosis induced by cisplatin and doxorubicin through activation of p38 MAPK. Biochem Biophys Res Commun 406: 299-304, 2011.

35. Chen R, Dai RY, Duan CY, Liu YP, Chen SK, Yan DM, Chen CN, Wei $\mathrm{M}$ and $\mathrm{Li} \mathrm{H}$ : Unfolded protein response suppresses cisplatin-induced apoptosis via autophagy regulation in human hepatocellular carcinoma cells. Folia Biol (Praha) 57: 87-95, 2011.

36. Rabik CA, Fishel ML, Holleran JL, Kasza K, Kelley MR, Egorin MJ and Dolan ME: Enhancement of cisplatin [cis-diammine dichloroplatinum (II)] cytotoxicity by O6-benzylguanine involves endoplasmic reticulum stress. J Pharmacol Exp Ther 327: 442-452, 2008.

37. Nawrocki ST, Carew JS, Pino MS, Highshaw RA, Dunner K Jr, Huang P, Abbruzzese JL and McConkey DJ: Bortezomib sensitizes pancreatic cancer cells to endoplasmic reticulum stress-mediated apoptosis. Cancer Res 65: 11658-11666, 2005.

38. Suzuki M, Endo M, Shinohara F, Echigo S and Rikiishi H: Enhancement of cisplatin cytotoxicity by SAHA involves endoplasmic reticulum stress-mediated apoptosis in oral squamous cell carcinoma cells. Cancer Chemother Pharmacol 64 1115-1122, 2009

39. Park IJ, Kim MJ, Park OJ, Choe W, Kang I, Kim SS and Ha J: Cryptotanshinone induces ER stress-mediated apoptosis in HepG2 and MCF7 cells. Apoptosis 17: 248-257, 2012.

40. Rovetta F, Stacchiotti A, Consiglio A, Cadei M, Grigolato PG, Lavazza A, Rezzani R and Aleo MF: ER signaling regulation drives the switch between autophagy and apoptosis in NRK-52E cells exposed to cisplatin. Exp Cell Res 318: 238-250, 2012. 